\title{
Hsp90 turns up the heat on Candida
}

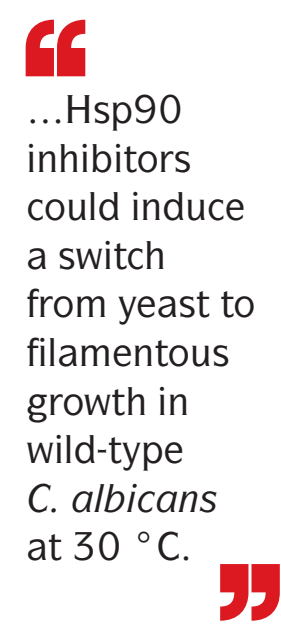

The molecular basis for the temperature dependence of the morphological switch from yeast to filamentous growth in Candida albicans is a longstanding puzzle. The answer lies in the molecular chaperone heat shock protein 90 (Hsp90), according to a recent report in Current Biology.

In C. albicans the transition from the yeast to the filamentous form is intimately associated with virulence. It has been known for many years that in vitro, environmental stresses such as nutrient limitation or the presence of serum induce this transition only at $37^{\circ} \mathrm{C}$, but the basis for this temperature dependence was unknown.

Hsp90 is the chaperone for many different proteins, including key regulators of eukaryotic cell signalling pathways. Rebecca Shapiro and colleagues discovered that C. albicans Hsp90 was involved in the morphogenetic switch when they noticed that Hsp90 inhibitors could induce a switch from yeast to filamentous growth in wild-type C. albicans at $30^{\circ} \mathrm{C}$. The authors suspected that the trigger for the morphological switch could be the relief of Hsp90-mediated repression, which they confirmed by showing that Hsp90 does not function effectively at the elevated temperatures that favour filamentation.

A screen to understand the mechanism of Hsp90-mediated repression implicated the Ras1-protein kinase A (PKA) signalling pathway. To determine where in this pathway Hsp90 acts, Shapiro et al. analysed the effects of the quorum sensing regulators farnesol and dodecanol, as both these compounds are thought to target the Ras1-PKA pathway at, or downstream of, Cdc35, which in turn is directly downstream of activated Ras1. Neither compound affected the Hsp90-mediated repression, which suggests that Hsp90 acts downstream of Cdc35 in the Ras1-PKA pathway.

Finally, Shapiro et al. found that depleting Hsp90 attenuated C. albicans virulence in a murine infection model. The authors conclude that targeting C. albicans Hsp90 "provides an attractive therapeutic strategy".

Sheilagh Molloy

ORIGINAL RESEARCH PAPER Shapiro, R. S. et al. Hsp90 orchestrates temperature-dependent Candida albicans morphogenesis via Ras1-PKA signalling. Curr. Biol. 19, 621-629 (2009) FURTHER READING Cowen, L. E. The evolution of fungal drug resistance: modulating the trajectory from genotype to phenotype. Nature Rev. Microbiol. 6, 187-198 (2008) 